
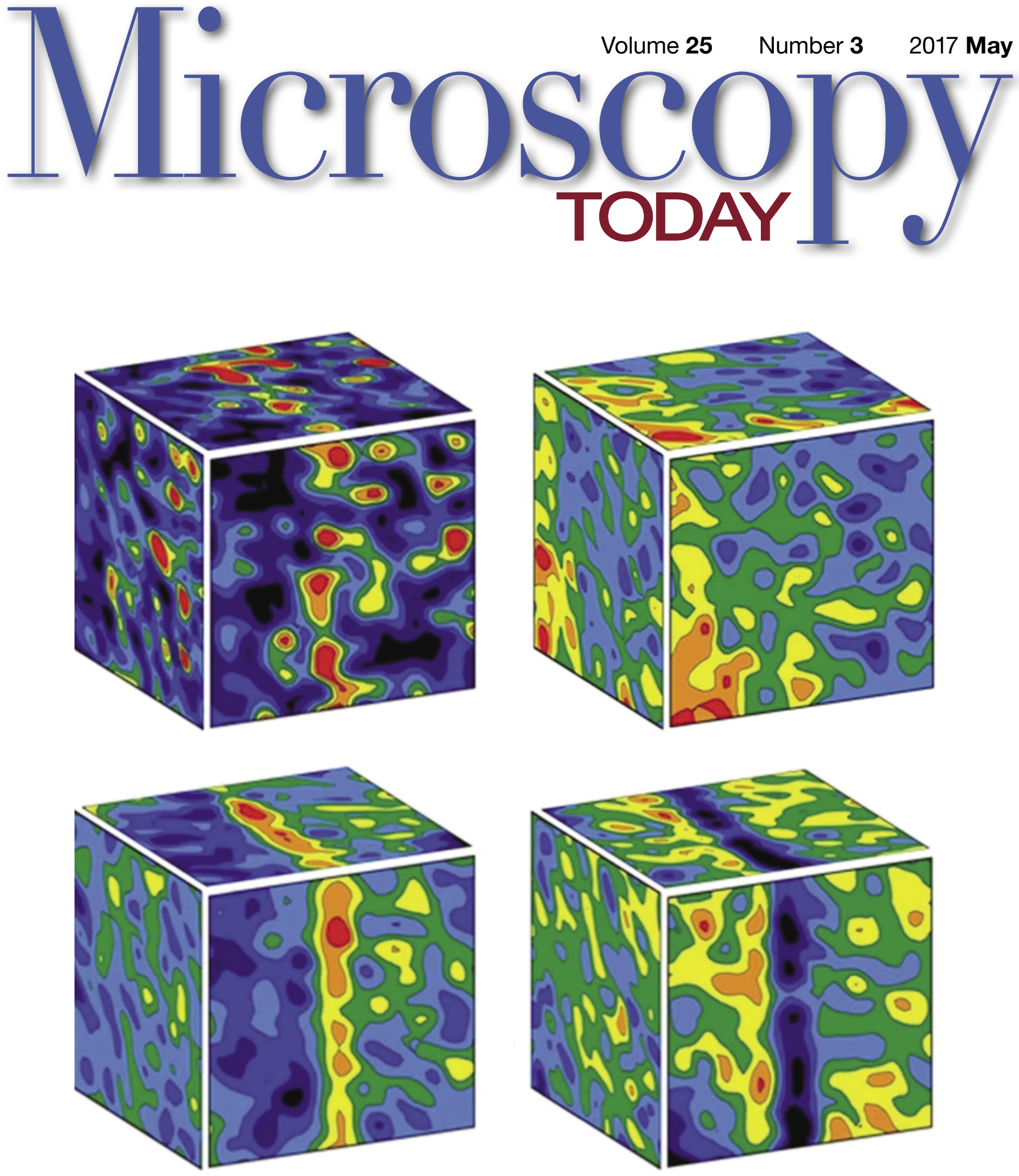


\title{
HITACHI
}

\section{Inspire the Next}

\section{The HF5000 $200 \mathrm{kV}$ Cold FEG \\ Aberration-Corrected TEM/STEM + SEM}

\section{The Innovative 200 kV TEM/STEM + SEM Is Here!}

\section{Three imaging modes (TEM/STEM + SEM) integrated into one system with automated aberration correction}

\begin{abstract}
The Hitachi HF5000 TEM/STEM + SEM with aberration-corrected STEM/SEM accommodates simultaneous acquisition of surface and transmission images at sub-atomic resolution. Surpassing traditional TEM/STEM imaging, the HF5000's proprietary SE capability can image surfaces of both transmissive and bulk samples while providing simultaneous EDS analysis. Hitachi's own highly automated and probe-forming aberration correction is designed specifically to make sub- $\AA$ imaging easy.

Our unique cold field-emission gun technology delivers high brightness, extended stability of the probe current, and leading-energy resolution without a monochromator. With the availability of dual X-ray detectors, we guarantee fast and highly sensitive EDS analyses. EELS and many other options are available.
\end{abstract}

For more details, contact us at: microscopy@hitachi-hta.com

\section{Think Outside the Lab}




\section{Don't miss}

special anniversary programming in honor of 75. 1942-2017 Microscopy Society of America and

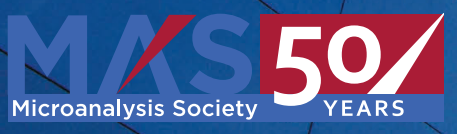

as well as the $50^{\text {th }}$ anniversary of the atom probe
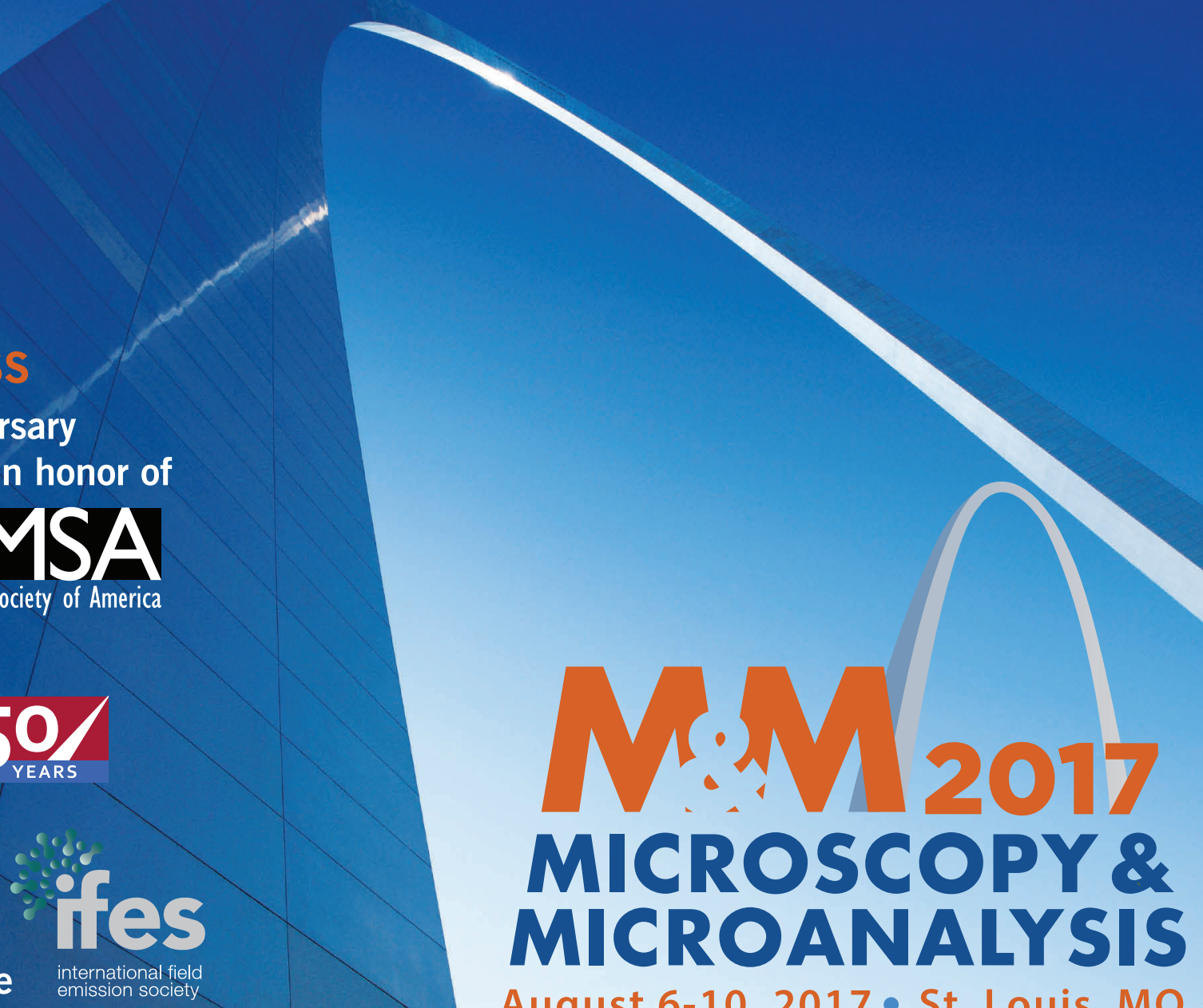

August 6-10, 2017 - St. Louis, MO

\section{http://www.microscopy.org/MandM/2017}

Listen to extraordinary plenary talks from Eric Betzig, Janelia Farm, and Keith Riles, University of Michigan

Attend one of FOUR stellar Pre-Meeting Congresses

Deep dive into specific topics in traditional day-long Sunday Short Courses

Experience hands-on demos for the latest microscopy products during Vendor Tutorials

Discuss recent work in depth with over 500 poster presenters

Visit the largest microscopy exhibit hall in the world with over 120 companies

Hear about cutting-edge scientific work in over 30 symposia in Physical, Biological, and Analytical Sciences

Network with colleagues and friends during happy hours and social events 


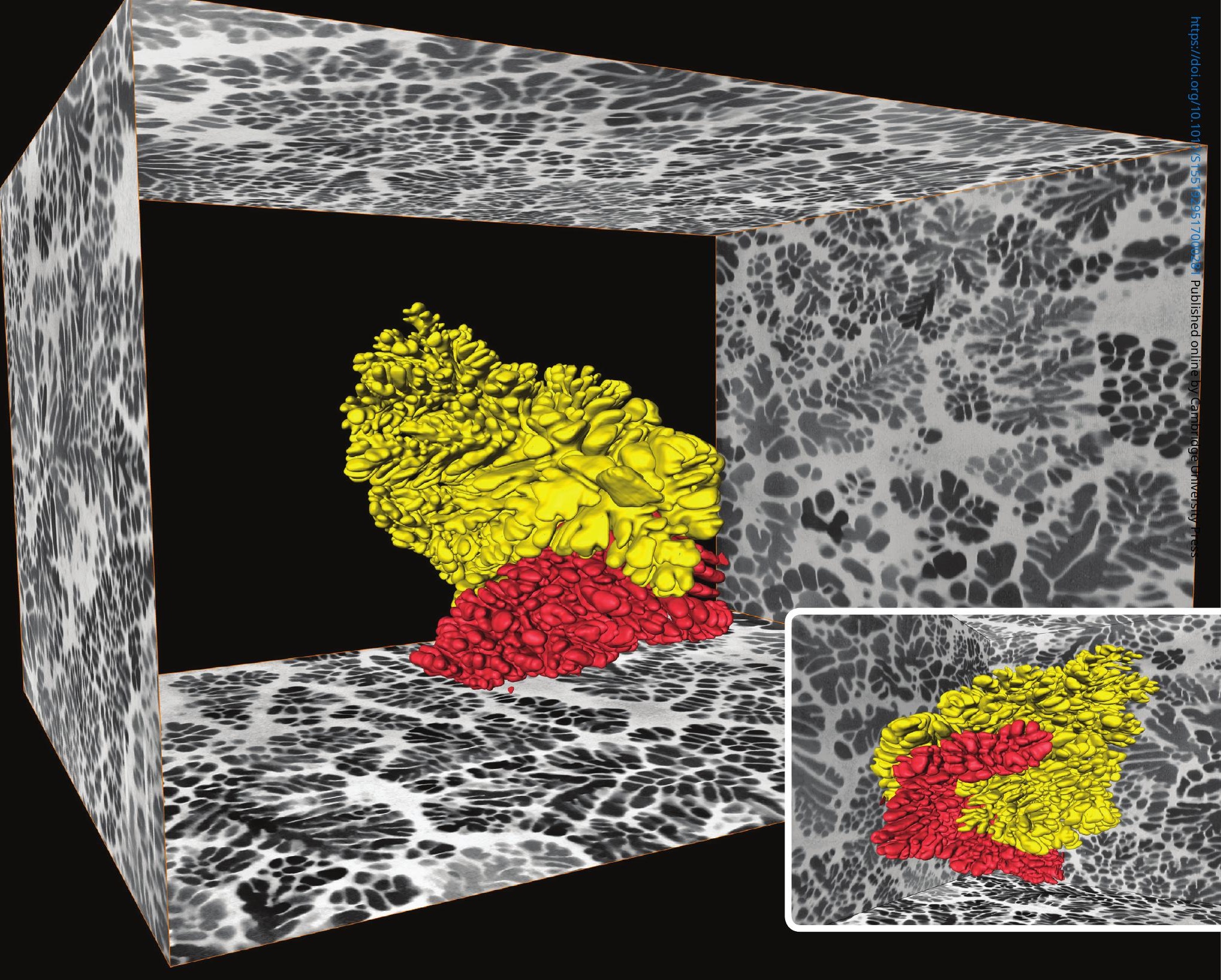

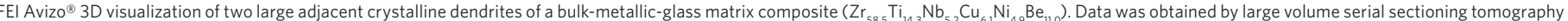
using the Helios PFIB DualBeam. The sectioned block is about $90 \times 80 \times 70 \mu \mathrm{m}^{3}$ Sample from The University of Tennessee USA. Images courtesy of The University of Manchester.

\section{Large 3D volumes with \\ unprecedented surface resolution}

Until recently, available technologies have limited the volumes and depths of materials that can be analyzed at high resolution, ultimately restricting the insight into structural, crystallographic, and chemical properties. This is no longer the case. The Helios ${ }^{\mathrm{TM}}$ PFIB DualBeam ${ }^{\mathrm{TM}}$ offers unrivaled access to regions of interest deep below the surface-combining serial section tomography with statistically relevant data analysis. 


\section{Contents}

About the Cover

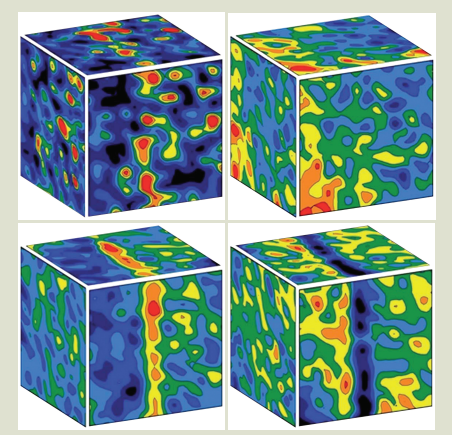

Atom probe concentration maps in the vicinity of a grain boundary in $\mathrm{Nd}$ doped ceria. Clockwise from upper left: $\mathrm{Al}, \mathrm{Ce}, \mathrm{O}$, and $\mathrm{Nd}$. Lowest concentration = black; highest concentration $=$ red . Width of cube $=16 \mathrm{~nm}$.

See article by Kelly and Panitz

\section{Microscopy}

\section{Atom Probe Tomography}

12 The First Fifty Years of Atom Probe

Thomas F. Kelly and John A. Panitz

\section{Scanning Electron Microscopy}

18 A Software Approach to Improving SEM Resolution, Image Quality, and Productivity

Eric Lifshin, Matthew Zotta, David Frey, Sarah Lifshin, Mandy Nevins, and Jeffrey Moskin

\section{Cryo-Electron Microscopy}

26 Charting Molecular Landscapes Using Cryo-Electron Tomography Laura Burbaum, Miroslava Schaffer, Benjamin D. Engel, Julia Mahamid, Sahradha Albert, Radostin Danev, Wolfgang Baumeister, and Jürgen M. Plitzko

\section{Lunar Materials}

32 Glassy with a Chance of Nanophase Iron: Space Weathering of Lunar Soil as Observed with Aberration-Corrected Scanning Transmission Electron Microscopy

K.D. Burgess and R.M. Stroud

\section{Microscopy 101}

40 Practical Solutions to Frequent Problems Encountered in Thin Sections Electron Microscopy

Manuela Lavorato and Clara Franzini-Armstrong

\section{Opinion}

46 A Call to Action Alwyn Eades

\section{Departments}

7 Editorial

8 Carmichael's Concise Review

48 Pioneers of Microscopy

50 Industry News

52 Product News
54 Microscopy and Microanalysis Highlights

56 NetNotes

61 Calendar of Meetings

65 Dear Abbe

66 Index of Advertisers 


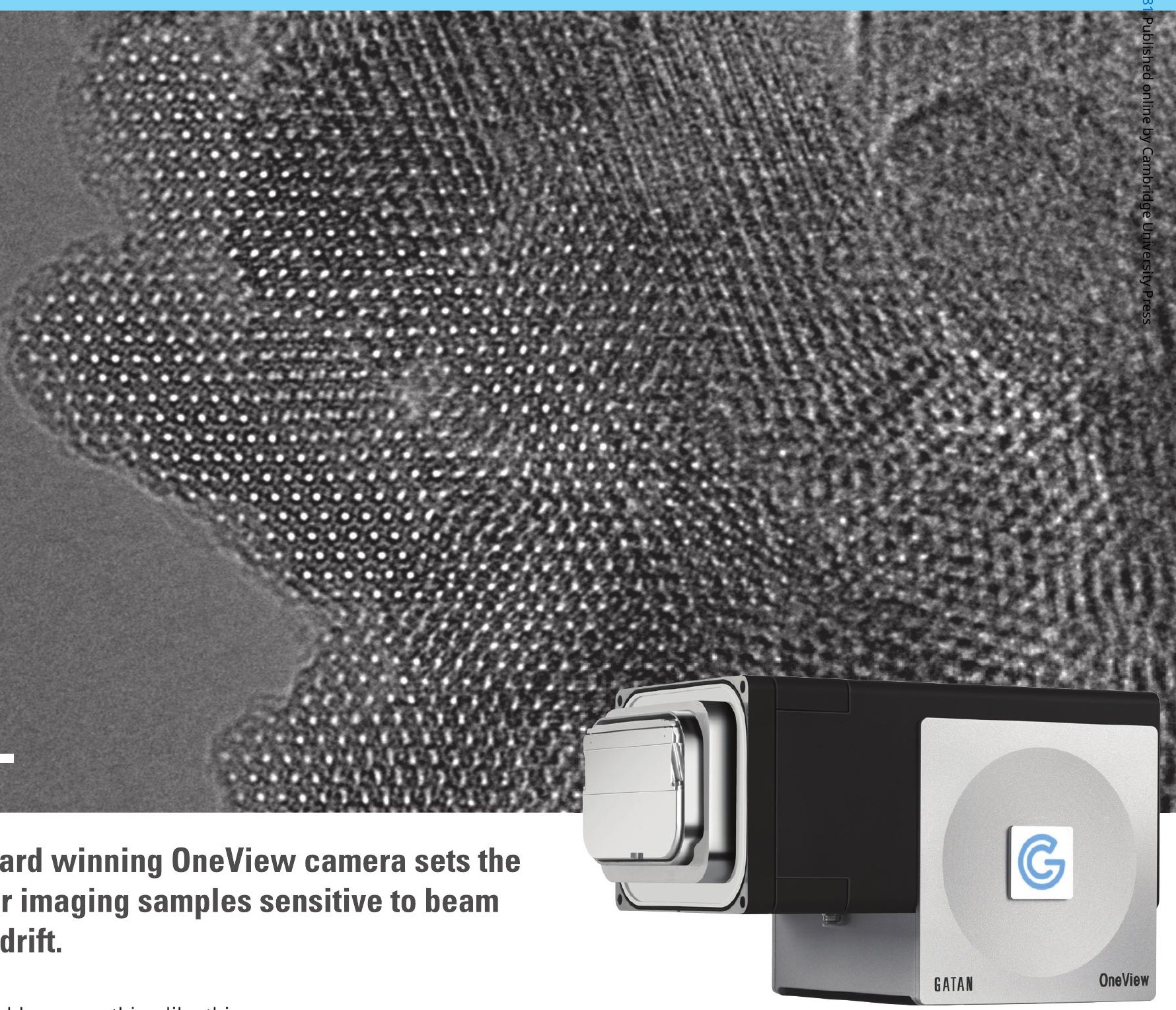

"My review would go something like this:

Awesome camera

Verified Purchase

This has been a great $4 k$ camera for low-dose imaging. The low-dose imaging capabilities of the camera and the option for drift correction work great together! To be honest, I assumed the drift correction option would be something that I would never use. ... In reality, I have gotten the best HRTEM images from my samples using the drift correction option. ... This camera has given new life to my elderly TEM!

\section{I would buy this camera again!"}

- Thomas M. Rea, Senior EM, 35 years

See how real-time drift correction allows you to record images before the sample stops drifting and guarantees the highest quality images at the lowest total dose, every time.

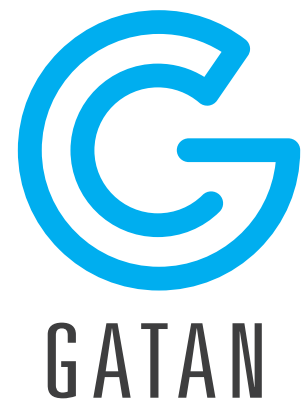

